\title{
Shape Analysis of Human Brain Interhemispheric Fissure Bending in MRI
}

\author{
Lu Zhao ${ }^{1}$, Jarmo Hietala ${ }^{2}$, and Jussi Tohka ${ }^{1}$ \\ 1 Department of Signal Processing, Tampere University of Technology, \\ FIN-33101, Tampere, Finland \\ lu.zhao@tut.fi \\ 2 Department of Psychiatry, University of Turku, FIN-20014, Turku, Finland
}

\begin{abstract}
This paper introduces a novel approach to analyze Yakovlevian torque by quantifying the bending of human brain interhemispheric fissure in three-dimensional magnetic resonance imaging. It extracts the longitudinal medial surface between the cerebral hemispheres, which are segmented with an accurate and completely automatic technique, as the shape representation of the interhemispheric fissure. The extracted medial surface is modeled with a polynomial surface through least-square fitting. Finally, curvature features, e.g. principal, Gaussian and mean curvatures, are computed at each point of the fitted medial surface to describe the local bending of the interhemispheric fissure. This method was applied to clinical images of healthy controls (12 males, 7 females) and never-medicated schizophrenic subjects (11 males, 7 females). The hypothesis of the normal interhemispheric fissure bending (rightward in the occipital region) was quantitatively demonstrated. Moreover, we found significant differences $(p<0.05)$ between the male schizophrenics and healthy controls with respect to the interhemispheric fissure bending in the frontal and occipital regions. These results show that our method is applicable for studying abnormal Yakovlevian torque related to mental diseases.
\end{abstract}

\section{Introduction}

The left and right hemispheres of human brain are roughly equal in volume, weight and density, however, the tissue distribution differs notably between the two hemispheres. Among the most prominent observations of structural asymmetry of human brain are the right frontal and left occipital petalias 1]. Petalias are the greater protrusion of one hemisphere relative to the other at the frontal and occipital regions.

The right frontal and left occipital petalias were revealed from computed tomography (CT) and magnetic resonance imaging (MRI) scans with width measurements [2]3. Bilder et al. 4] conducted the first volumetric study for petalias in MRI using manual outlining of lobar volumes. Recently, automatic image analysis methods have been applied to investigate the petalias of human brain in MRI with respect to local volumetric asymmetry. The voxel-wise interhemispheric differences in tissue volume [5] and tissue density [6] was studied using the 
reflection-based registration. In [7] and [8], the reflection method was extended to produce low dimensional maps showing interhemispheric differences in tissue volume in an array of column orthogonal to the mid-plane (two-dimensional) and profiles of coronal slice volumes (one-dimensional). Thirion et al. [9] quantified the regional volume differences between homologous structures in opposite brain hemispheres by nonrigidly co-registering the two hemispheres with each other.

Another prominent geometric distortion of the brain hemispheres, known as Yakovlevian torque, is that the right frontal lobe is torqued forward the left, and the left occipital lobe extends across the midline (over the right occipital lobe) and skews the interhemispheric fissure towards the right [1. In this work, we developed an automatic shape analysis method to analyze the interhemispheric fissure bending of human brain in three-dimensional (3D) MRI using curvature features. This method can provide morphological interpretations of brain asymmetry that are easy to understand. To our knowledge, no previous studies of Yakovlevian torque by quantifying the interhemispheric fissure bending exist.

\section{Methods}

\subsection{Image Preprocessing}

The cerebral hemispheres $(\mathrm{CH})$ are extracted and segmented in MR brain images in the acquisition space by using an automatic $\mathrm{CH}$ segmentation technique [1011. For inter-subject comparisons, the segmented $\mathrm{CH}$ volumes are normalized into the ICBM152 space (dimension: $91 \times 109 \times 91$ voxels, voxel size: $2 \times 2 \times 2$ $\mathrm{mm}^{3}$ ) [12] with a 12-parameter affine transformation in SPM5 [13].

\subsection{Representation of the Interhemispheric Fissure Shape}

The interhemispheric fissure of human brain refers to the narrow groove separating the left and right $\mathrm{CH}$. We use a medial interhemispheric surface to represent the shape of the interhemispheric fissure. Denote the lateral, longitudinal and vertical axes of the image space as $X, Y$ and $Z$, respectively, the normalized segmented left and right $\mathrm{CH}$ as $\mathrm{lCH}$ and $\mathrm{rCH}$, the Euclidean distances from a image voxel $(x, y, z)$ to $l C H$ and $r C H$ as $D_{l}(x, y, z)$ and $D_{r}(x, y, z)$. We define the medial interhemispheric surface as a longitudinal surface $S$ where each surface point is located with its projection $(y, z)$ on the $Y Z$ plane. The lateral magnitude $x_{S}$ of $S$ at $(y, z)$ is found as

$$
x_{S}(y, z)=\arg \min _{x}\left\{\left|D_{l}(x, y, z)-D_{r}(x, y, z)\right|\right\} .
$$

To build a mathematical model of $S$, a two-variable polynomial of degree $k$

$$
\hat{x}_{S}(y, z)=\sum_{i=0}^{k} \sum_{j=0}^{i} a_{i j} y^{i-j} z^{j}
$$

is used to fit it, where $\hat{x}_{S}$ is the approximation of the lateral magnitude $x_{S}$ at $(y, z)$. Because the curvature features will be computed based on the second 
fundamental form of the fitted surface, the polynomial must be two-times differentiable, i.e. $k \geq 2$. In addition, $k$ should not be very large, since the fitted surface needs to be smooth enough to present the global bending tendency of the interhemispheric fissure. The coefficients $a_{i j}$ are estimated with the least-square fitting approach. In the image space, let $\mathbf{x}_{S}$ be the column vector containing the $x_{S}$ values of all surface points, $B$ be the matrix whose each row consists of the values of item $y^{i-j} z^{j}$ in Eq2 for all $(y, z)$. The least-square solutions of $a_{i j}$, written in a column vector $\mathbf{a}$, are

$$
\mathbf{a}=\left(B^{T} B\right)^{-1} B^{T} \mathbf{x}_{S} .
$$

\subsection{Curvature Feature Computation}

We compute the $2 \times 2$ Hessian matrix

$$
\mathcal{H}=\left(\begin{array}{ll}
\frac{\partial^{2} \hat{x}_{S}}{\partial y^{2}} & \frac{\partial^{2} \hat{x}_{S}}{\partial y \partial z} \\
\frac{\partial^{2} \hat{x}_{S}}{\partial y \partial z} & \frac{\partial^{2} \hat{x}_{S}}{\partial z^{2}}
\end{array}\right),
$$

based on the approximation (Eq,2) of $S$. Through $\mathcal{H}$, a number of curvature features can be computed at each point of $S$. The two eigenvalues of $\mathcal{H}, \kappa_{1}$ and $\kappa_{2}$, are the principal curvatures, which describe the maximum and minimum curvatures. The tangent directions of the principal curvatures, called principal directions, are given by the orthogonal eigenvectors of $\mathcal{H}$. The Gaussian curvature $K=\kappa_{1} \kappa_{2}$ and mean curvature $H=\left(\kappa_{1}+\kappa_{2}\right) / 2$. The diagonal elements of $\mathcal{H}, C_{X Y}=\frac{\partial^{2} \hat{x}_{S}}{\partial y^{2}}$ and $C_{X Z}=\frac{\partial^{2} \hat{x}_{S}}{\partial z^{2}}$, are the curvatures in $X Y$ and $X Z$ planes, respectively. The above curvature features, except Gaussian curvature and principal directions, are taken to be positive if the relative curve turns in the same direction as the surface's chosen normal, and otherwise negative.

We also define the integrated average of the curvature features in regions of interest (ROIs). ROIs were extracted by masking $S$ with the projection of the LONI Probabilistic Atlas (LPBA40) [14] on its mid-sagittal plane. The employed version of LPBA40 is LPBA40/SPM5, which was constructed by transforming the manual delineations into the ICBM152 template where the longitudinal median plane is the mid-sagittal plane. Because the image containing the normalized $\mathrm{CH}$ volumes is digital and has voxel size of $2 \times 2 \times 2 \mathrm{~mm}^{3}$, the projection of each ROI on the mid-sagittal plane consists of a number of square cells with size of $2 \times 2 \mathrm{~mm}^{2}$. Thus, the integrated average $\xi_{f}$ of a curvature feature $f$ in a ROI is

$$
\xi_{f}=\frac{\sum_{c_{i}} \int_{y_{i}^{0}}^{y_{i}^{0}+2} \int_{z_{i}^{0}}^{z_{i}^{0}+2} f(y, z) \sqrt{\left(\frac{\partial \hat{x}_{S}}{\partial y}\right)^{2}+\left(\frac{\partial \hat{x}_{S}}{\partial z}\right)^{2}+1} d y d z}{\sum_{c_{i}} \int_{y_{i}^{0}}^{y_{i}^{0}+2} \int_{z_{i}^{0}}^{z_{i}^{0}+2} \sqrt{\left(\frac{\partial \hat{x}_{S}}{\partial y}\right)^{2}+\left(\frac{\partial \hat{x}_{S}}{\partial z}\right)^{2}+1} d y d z},
$$

where $c_{i}=\left[y_{i}^{0}, y_{i}^{0}+2\right) \times\left[z_{i}^{0}, z_{i}^{0}+2\right)$ is a single cell in the projection of the ROI on the mid-sagittal plane; $y_{i}^{0}$ and $z_{i}^{0}$ are the $Y$ and $Z$ coordinates of the origin of $c_{i}$; the numerator is the total value of $f$ in the ROI; and the denominator is the total area of the ROI. In this work, the numerical integrations in $\mathrm{Eq} 5$ were solved with the two-dimensional Simpson's rule [15]. 


\subsection{Statistical Shape Analysis}

We introduce two alternative ways to conduct the statistical shape analysis of the curvature features of the interhemispheric surface between two subject populations. The first is point-to-point analysis. The average of the normalized segmented $\mathrm{CH}$ volumes of all studied subjects is computed. The projection of this averaged volume on the ICBM152 template's mid-sagittal plane are used to locate the points of interest (POIs) on the fitted medial interhemispheric surface. Because the probability distribution types of the curvature features are unknown, the nonparametric Wilcoxon Rank Sum test is used to assess the differences in the curvature features between two populations at each POI. The second way is ROI-to-ROI analysis. The computation of the integrated average curvatures in ROIs includes a large amount of integration (see Eq.5). Therefore, due to the central limit theorem, we can assume that the integrated average curvatures are approximately Gaussian distributed. For each ROI, the integrated average curvatures are analyzed between groups with t-test. For both the point-to-point and ROI-to-ROI analyses, the significance level is set to 0.05 .

\section{$3 \quad$ Experiments and Results}

\subsection{Materials}

The proposed method was applied to clinical T1-weighted MR images (voxel size: $1.5 \times 1.5 \times 1.0 \mathrm{~mm}^{3}$; dimension: $256 \times 256 \times 150$ or $256 \times 256 \times 170$ voxels $)$ of 18 schizophrenic subjects (11 males, 7 females) and 19 healthy controls (12 males, 7 females) [16. All the subjects were right-handed. The schizophrenic patients were never-medicated, i.e. the brain asymmetry patterns in these subjects were not affected by antipsychotic drugs.

\subsection{Quantification for Interhemispheric Fissure Bending}

The degree of the polynomial used for surface fitting (Eq2) was 4, because it is the lowest degree for the curvature features, computed based on the second fundamental form of the fitted surface, to remain nonlinear w.r.t to $(y, z)$. Employing polynomial surfaces with a higher degree would produce more accurate surface fitting, but it would also produce non-essential shape information (noise) for analyzing the interhemispheric fissure bending. In MRI, the laterally-oriented interhemispheric fissure bending caused by Yakovlevian torque is mainly observed in the transverse view ( $X Y$ planes), and the geometric interpretations of the Gaussian and principal curvatures are complicated. Therefore, we only considered the mean curvature $H$ and the curvature in the $X Y$ plane $C_{X Y}$ in the presented experiments. Because the projections of chosen normals on $X$ axis at each point of the fitted medial interhemispheric surface were always rightward, positive values of $H$ and $C_{X Y}$ indicated laterally rightward bending, and negative values indicated leftward bending. Figs 11 and 2 illustrate medial interhemispheric surface extraction, fitting and the corresponding $H$ and $C_{X Y}$ values at POIs. 


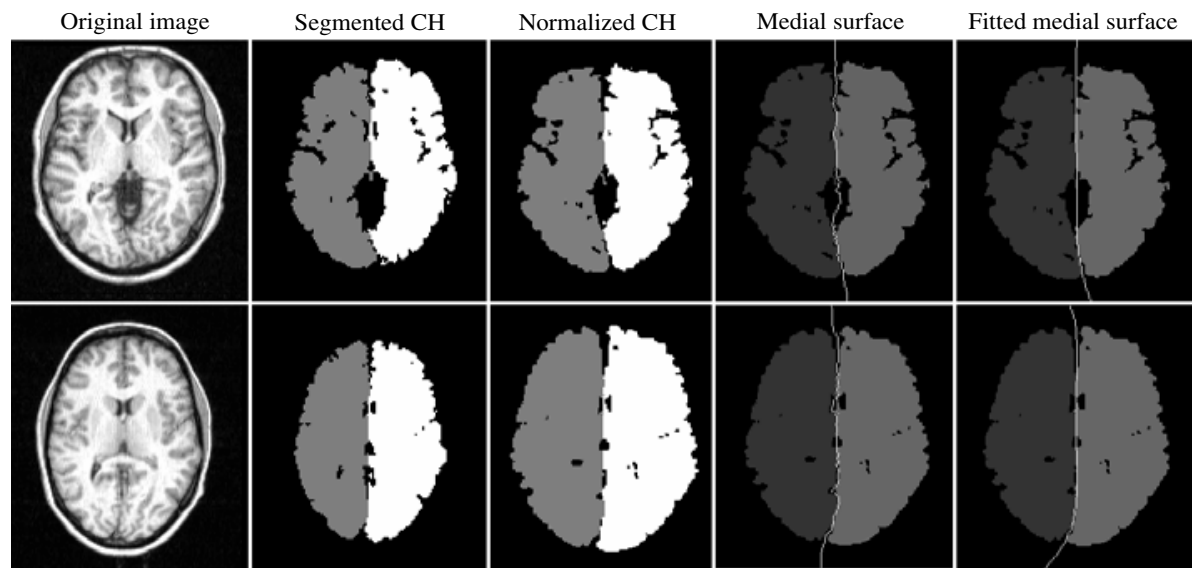

Fig. 1. Medial interhemispheric surface extraction and fitting. The first and second rows respectively show examples for subjects with normal (rightward) and abnormal (leftward) interhemispheric fissure bending. Original images were in neurological convention, namely the left (or right) hemisphere was in the left (or right). The extracted medial interhemispheric surfaces and fitted surfaces are visualized as longitudinal lines in the transverse view.
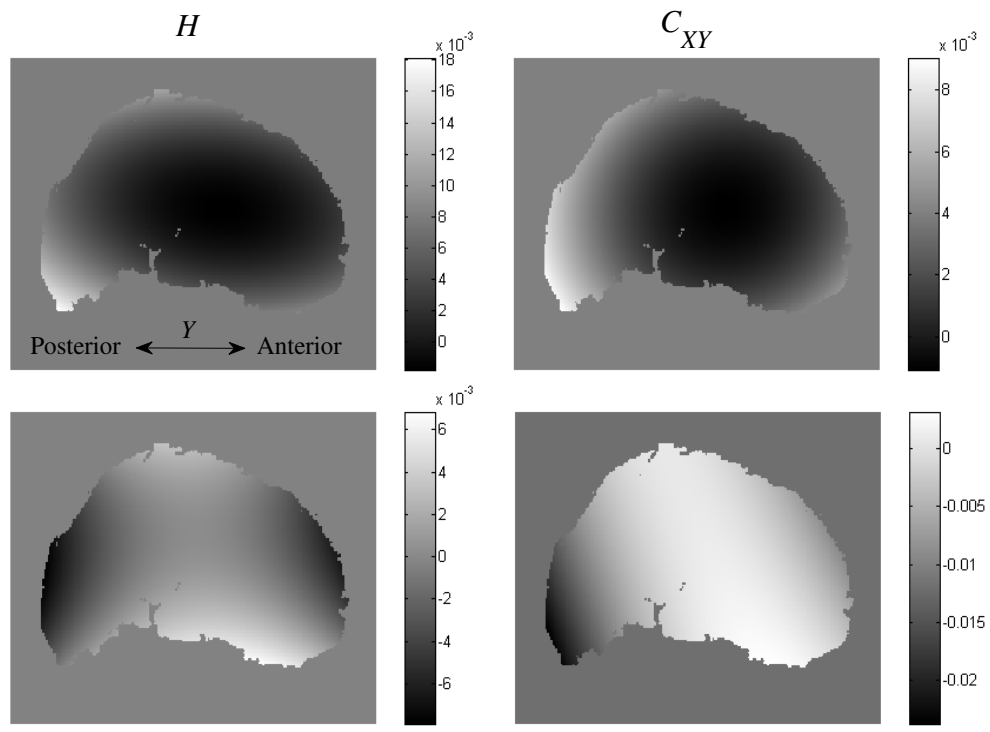

Fig. 2. Visualization in the sagittal view ( $Y Z$ plane) for the values of $H$ and $C_{X Y}$ at POIs. The first and second rows respectively correspond to the subjects shown in the first and second rows in Fig 1 . 
Table 1. Means of $\xi_{H}$ and $\xi_{C_{X Y}}$ in the frontal and occipital regions for male controls (MC), female controls (FC), male schizophrenics (MS), and female schizophrenics (FS)

\begin{tabular}{|c|c|c|c|c|}
\hline & MC & FC & MS & FS \\
\hline & \multicolumn{4}{|c|}{ Frontal region } \\
\hline mean of $\xi_{H}$ & 7.8 e- 4 & -2.8 e- 4 & -8.2 e- 4 & 7.5 e-5 \\
\hline mean of $\xi_{C_{X Y}}$ & 7.5 e- 4 & -3.4 e- 4 & -5.3 e- 4 & 3.9 e-4 \\
\hline & \multicolumn{5}{|c|}{ Occipital region } \\
\hline mean of $\xi_{H}$ & 1.8 e-3 & 2.4 e- 4 & 8.9 e- 4 & 8.3 e-4 \\
\hline mean of $\xi_{C_{X Y}}$ & 2.6 e-3 & 9.6 e- 4 & 1.1 e-3 & 1.6 e-3 \\
\hline
\end{tabular}

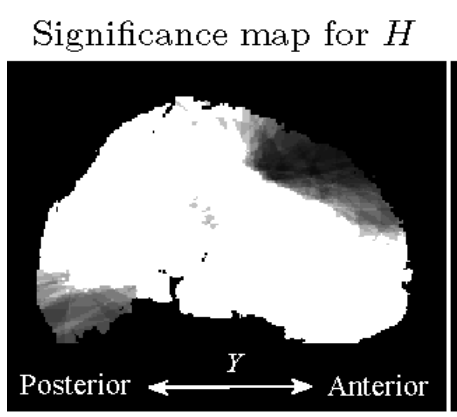

Significance map for $C_{X Y}$
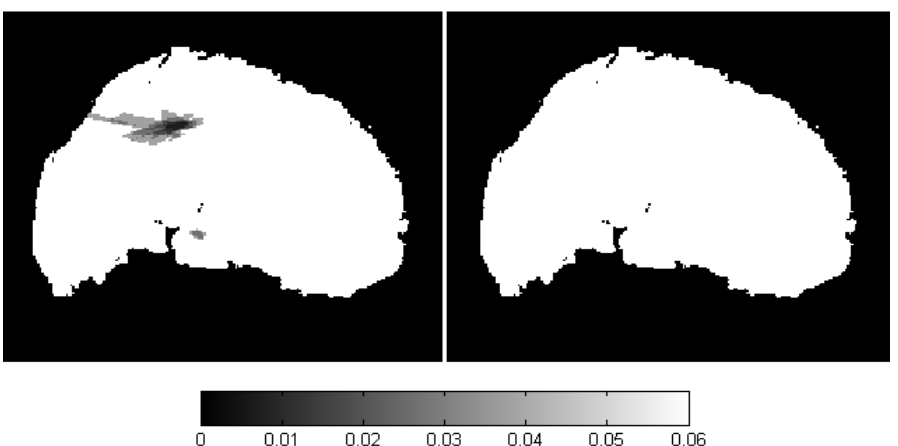

Fig. 3. Statistically significant results of point-to-point analysis (uncorrected $p<0.05$ ) for comparison between controls and schizophrenics with respect to $H$ and $C_{X Y}$. The first and second rows respectively present the results for males and females.

We calculated the integrated average values of $H$ and $C_{X Y}$, denoted as $\xi_{H}$ and $\xi_{C_{X Y}}$, in ROIs corresponding to the frontal and occipital regions. For every subject, the accuracy of the proposed method to automatically detect the lateral direction of interhemispheric fissure bending with curvature features was evaluated by comparing the bending direction indicated by the sign of $\xi_{H}$ or $\xi_{C_{X Y}}$ in the occipital region against the bending direction manually identified in the transverse slices of the original image. For all the 37 studied subjects, the proposed method obtained correct detection for 34 subjects with $\xi_{H}$ and for 36 
subjects with $\xi_{C_{X Y}}$. The means of $\xi_{H}$ and $\xi_{C_{X Y}}$ for different populations are tabulated in Table11. In all populations, the means of $\xi_{H}$ and $\xi_{C_{X Y}}$ in the occipital region were positive, and their absolute values were always notably greater than their counterparts in the frontal region. This indicates that, in average, the interhemispheric fissure bending of right-handed subject mainly occurs in the occipital region and is laterally rightward. This re-confirms the hypothesis of interhemispheric fissure bending caused by normal Yakovlevian torque [1].

\subsection{Statistical Shape Analysis between Controls and Schizophrenics}

The results of statistical comparison between controls and schizophrenics with point-to-point analysis are shown in Fig 3. It can be seen that, for males, POIs of the significant difference between controls and schizophrenics were mainly located in the superior frontal region for $H$ and $C_{X Y}$, and in the inferior occipital region for $H$. Pointwise differences between female controls and schizophrenics were not as great as in males. From the ROI-to-ROI analysis, we found significant difference between male controls and schizophrenics with respect to the integrated average curvatures $\xi_{H}(p=0.0084)$ and $\xi_{C_{X Y}}(p=0.036)$ in the frontal region. This finding is well in line with above results of point-to-point analysis.

\section{Conclusion}

The traditional methods based on width and volume measurements have limited capabilities to quantify brain asymmetry in neuroimages. In this paper, we developed a novel method to automatically quantify the interhemispheric fissure bending caused by Yakovlevian torque with curvature features in 3D MRI. This method is applicable for making inferences on individual subjects as well as subject populations. In the application of the proposed method to a clinical data set containing MR images of healthy controls and never-medicated schizophrenics, the hypothesis of normal rightward interhemispheric fissure bending was quantitatively confirmed with the extracted curvature features. With the statistical analysis of the curvature features, the interhemispheric fissure bending of male schizophrenics was found to be significantly different from male controls' in the frontal and occipital regions.

Acknowledgements. This work was supported by the Academy of Finland, (application number 129657, Finnish Programme for Centres of Excellence in Research 2006- 2011), and the STATCORE cluster of the University Alliance Finland. Thanks to Dr. Saku Suuriniemi for help with numerical analysis.

\section{References}

1. Toga, W., Thompson, P.: Mapping brain asymmetry. Nature Reviews Neuroscience 4(1), 37-48 (2003)

2. LeMay, M., Kido, D.: Asymmetries of the cerebral hemispheres on computed tomograms. J. Comput. Assist. Tomogr. 2, 471-476 (1978) 
3. Kertesz, A., Polk, M., Black, S., Howell, J.: Sex, handedness, and the morphometry of cerebral asymmetries on magnetic resonance imaging. Brain Res. 530, 40-48 (1990)

4. Bilder, R., Wu, H., Bogerts, B., Degreef, G., Ashtari, M., Alvir, J., Snyder, P., Lieberman, J.: Absence of regional hemispheric volume asymmetries in first-episode schizophrenia. Am. J. Psychiatry 151(10), 1437-1447 (1994)

5. Good, C., Johnsrude, I., Ashburner, J., Henson, R., Friston, K., Frackowiak, R.: Cerebral asymmetry and the effects of sex and handedness on brain structure: a voxel-based morphometric analysis of 465 normal adult human brains. NeuroImage $14,685-700(2001)$

6. Watkins, K., Paus, T., Lerch, J., Zidjenbos, A., Collins, D., Neelin, P., Taylor, J., Worsley, K., Evans, A.: Structural asymmetries in the human brain: a voxel-based statistical analysis of 142 MRI scans. Cereb. Cortex 11, 868-877 (2001)

7. Mackay, C., Barrick, T., Roberts, N., DeLisi, L., Maes, F., Vandermeulen, D., Crow, T.: Application of a new image analysis technique to the study of brain asymmetry in schizophrenia. Psychiatry Res. NeuroImaging 124(1), 25-35 (2003)

8. Barrick, T., Mackay, C., Prima, S., Maes, F., Vandermeulen, D., Crow, T., Roberts, N.: Automatic analysis of cerebral asymmetry: an exploratory study of the relationship between brain torque and planum temporale asymmetry. NeuroImage 24(3), 678-691 (2005)

9. Thirion, J.P., Prima, S., Subsol, G., Roberts, N.: Automatic analysis of cerebral asymmetry. Med. Image Anal. 4, 111-121 (2001)

10. Zhao, L., Tohka, J., Ruotsalainen, U.: Accurate 3D left-right brain hemisphere segmentation in MR images based on shape bottlenecks and partial volume estimation. In: Ersbøll, B.K., Pedersen, K.S. (eds.) SCIA 2007. LNCS, vol. 4522, pp. 581-590. Springer, Heidelberg (2007)

11. Zhao, L., Tohka, J.: Automatic compartmental decomposition for 3D MR images of human brain. In: 30th Annual International Conference of the IEEE Engineering in Medicine and Biology Society, Vancouver, Canada, pp. 3888-3891 (2008)

12. Evans, A., Collins, D., Neelin, P., MacDonald, D., Kamber, M., Marrett, T.: Threedimensional correlative imaging: applications in human brain mapping. In: Huerta, M. (ed.) Functional Neuroimaging: Technical Foundations, pp. 145-162. Academic Press, San Diego (1994)

13. Ashburner, J., Friston, K.: Nonlinear spatial normalization using basis functions. Human Brain Mapping 7(4), 254-266 (1999)

14. Shattuck, D., Mirza, M., Adisetiyo, V., Hojatkashani, C., Salamon, G., Narr, K., Poldrack, R., Bilder, R., Toga, A.: Construction of a 3D probabilistic atlas of human cortical structures. NeuroImage 39, 1064-1080 (2008)

15. Süli, E., Mayers, D.: An Introduction to Numerical Analysis. Cambridge University Press, Cambridge (2003)

16. Laakso, M., Tiihonen, J., Syvälahti, E., Vilkman, H., Laakso, A., Alakare, B., Räkköläinen, V., Salokangas, R., Koivisto, E., Hietala, J.: A morphometric MRI study of the hippocampus in first-episode, neuroleptic-naive schizophrenia. Schizophr Res. 50(1-2), 3-7 (2001) 